\title{
POLYVINYLPYRROLIDONE-BASED COATINGS FOR POLYURETHANES - THE EFFECT OF REAGENT CONCENTRATION ON THEIR CHOSEN PHYSICAL PROPERTIES
}

\author{
Beata Butruk*, Maciej Trzaskowski, Tomasz Ciach \\ Warsaw University of Technology, Faculty of Chemical and Process Engineering, Department of \\ Biotechnology and Bioprocess Engineering, Waryńskiego 1, 00-645 Warszawa, Poland
}

\begin{abstract}
A method of manufacturing hydrogel coatings designed to increase the hydrophilicity of polyurethanes (PU) is presented. Coatings were obtained from polyvinylpyrrolidone (PVP) by free radical polymerisation. The authors proposed a mechanism of a two-step grafting - crosslinking process and investigated the influence of reagent concentration on the coating's physical properties hydrogel ratio $(H G)$ and equilibrium swelling ratio $(E S R)$. A surface analysis of freeze-dried coatings using scanning electron microscopy (SEM) showed a highly porous structure. The presented technology can be used to produce biocompatible surfaces with limited protein and cell adhesive properties and can be applied in fabrication of number of biomedical devices, e.g. catheters, vascular grafts and heart prosthesis.
\end{abstract}

Keywords: polyurethanes, surface modification, surface hydrophilization, hydrogel

\section{INTRODUCTION}

Superhydrophilic surfaces are built of highly hydrophilic polymer chains. Chains are bound to the implant surface in a brush-like manner. In an aqueous medium a significant amount of water is bound between polymer chains (Kim, 2002). Such a surface, consisting of over $90 \%$ of water, has very low surface energy, which ensures low protein adsorption adhesion and prevents cell adhesion (Le and Scott, 2010). If a surface is thick enough, proteins protruding from macrophage's surface do not reach polymer matrix. For instance, a macrophage's membrane protein CD4 protrudes $14 \mathrm{~nm}$ over the cell membrane, while a hydrophilic polymer brush is usually tens of nanometres long (Le and Scott, 2010). Hydrogel polymer surfaces do not activate the immune system, which is a crucial factor in terms of implantable devices (Xu and Siedlecki, 2007). Surfaces of highly water-binding polymers, e.g. polysaccharides and polysaccharide acids, polyethers, polyalcohols and others may be obtained. Negative charge, typical for animal tissues, is favourable. In practice, hyaluronic acid, heparin, polyethylene glycol and polyvinyl alcohol are usually used. Long-lasting implants must be resistant to enzymes and oxidative agents from blood. Polyvinylpyrrolidone (PVP) seems to be a suitable material for this purpose. PVP is characterised by high water-binding properties, high biocompatibility and resistance to mammalian enzymatic apparatus and oxidation (Patel and Mequanint, 2007).

Various methods of coating a polymer with a layer of another polymer are known. Generally, physical and chemical methods can be distinguished. Coatings of various thicknesses are obtained easily by most commonly used physical methods. Coatings are bound to the matrix by mechanical forces, Van der Waals forces or polymer chains entanglement. The binding is facilitated by the presence of a 
solvent or higher temperature, which relaxes polymer structure. More sophisticated coatings with a covalently bound polymer network are obtained by chemical methods. These coatings reveal better adhesion to the substrate material and their structure may be precisely designed and produced, regarding both the chemical and physical structure of a polymer network.

In this paper the authors present a new method to produce a highly hydrophilic, hydrogel coating for polymer substrates. The presented method is based on free-radical macromolecular polymerisation grafting. Thanks to the combination of slightly water-soluble peroxide absorbed on the substrate and a quick Fenton process (Walling et al., 1974), free radicals are formed mainly on the polymer surface. This assures formation of covalent bonds between polymer substrate and the coating together with a high overall grafting efficiency. The presented coatings are designed to improve hydrophilicity of medical grade polyurethanes (PUs) and can be applied in medical devices that have permanent longterm contact with patients' tissues. In the previous paper (Butruk et al., 2012) we demonstrated that PVP-based coating significantly improves PU hemocompatibility, which makes the material a potential candidate for use in cardiovascular applications. The aim of this study was to investigate the influence of reagent concentration on the chosen physical properties of the hydrogel layer and to propose the grafting's mechanism. We also assessed the quality of the fabricated coating in terms of coating's smoothness, homogeneity and durability.

\section{EXPERIMENTAL}

\subsection{Materials}

Polyurethane (Chronathane ${ }^{\mathrm{TM}} \mathrm{P}-75 \mathrm{~A}$, CardioTech) in a form of discs (40 $\mathrm{mm}$ in diameter, $2 \mathrm{~mm}$ thick) was used as a polymer substrate for further modifications. Reagents, namely PVP powder with average molecular weight of $360 \mathrm{kDa}$ (PVP360), iron (II) chloride (FeCl2), ascorbic acid (AA), cumene hydroperoxide (CHP), ethylene glycol dimethacrylate (EGDMA) and sodium dodecyl sulfate (SDS) were obtained from Sigma-Aldrich. Toluene was purchased from Chempur, Poland. Phosphatebuffered saline (PBS) was prepared by dissolving $8 \mathrm{~g}$ of $\mathrm{NaCl}, 0.2 \mathrm{~g}$ of $\mathrm{KCl}, 1.44 \mathrm{~g}$ of $\mathrm{Na}_{2} \mathrm{HPO}_{4}$ and $0.24 \mathrm{~g}$ of $\mathrm{KH}_{2} \mathrm{PO}_{4}$ in $1 \mathrm{~L}$ of distilled water, $\mathrm{pH}$ was adjusted to 7.4 .

\subsection{The surface modification of polyurethane}

Hydrogel coatings of PU discs were fabricated in a two-step dip-coating method as described elsewhere (Butruk et al, 2012). Briefly, the PU discs were immersed in a toluene solution containing given amounts of EGDMA and CHP for 10 minutes at $25^{\circ} \mathrm{C}$. The samples were then placed in a water solution containing given amounts of PVP, $\mathrm{FeCl}_{2}$ and $0.1 \%(\mathrm{w} / \mathrm{v}) \mathrm{AA}$ for 15 minutes at $25^{\circ} \mathrm{C}$. After the coating procedure, the polymer discs were washed with $0.1 \%(\mathrm{w} / \mathrm{v})$ SDS water solution for 5 minutes, followed by washing in water (overnight) and lyophilisation.

\subsection{Surface characterisation}

The materials coated with hydrogel were characterised by the following parameters: the equilibrium swelling ratio $(E S R)$ and the mass of hydrogel grafted to the surface per surface area $(H G)$. Both, ESR and $H G$ were determined gravimetrically using analytical scale (Mettler Toledo) with accuracy 0.0001g. ESR was studied according to ASTM D570 as follows: samples in the form of discs $(60 \mathrm{~mm}$ in diameter) were dried at $50{ }^{\circ} \mathrm{C}$ until a constant weight was reached and weighed. Next, the samples were placed in PBS solution at $37{ }^{\circ} \mathrm{C}$ and weighed at different intervals of time until equilibrium swelling was reached. ESR was calculated according to the formula (1), where $W_{e q}$ is the weight of a 
swollen sample at equilibrium, $W_{d}$ is the weight of a dry sample and $W_{0}$ is the initial weight of an unmodified dry PU disc. $H G$ was calculated according to the formula (2), where $W_{0}$ is the initial weight of an unmodified dry PU disc, $W_{d}$ is the weight of a dry PU disc covered with hydrogel layer and $S$ is the area of a PU disc. Samples for ESR and $H G$ measurements were prepared in triplicates.

$$
\begin{gathered}
E S R=\frac{W_{e q}-W_{d}}{W_{d}-W_{0}} \cdot 100 \% \\
H G=\frac{W_{d}-W_{0}}{S}
\end{gathered}
$$

The morphology of hydrogel layers was examined using Scanning Electron Microscopy (Phenom, FEI). Small specimens of the samples were immersed in distilled water for $24 \mathrm{~h}$. To conserve the structure of hydrogel without collapsing, the samples were kept in the temperature of $-40^{\circ} \mathrm{C}$ for $4 \mathrm{~h}$ and then freeze dried under vacuum. Finally, the samples were sputtered with gold and the microstructure of hydrogels was depicted.

\section{RESULTS AND DISCUSSION}

\subsection{Mechanism of the coating process}

Although the presented process is based on a free radical polymerisation, the standard radical-based grafting procedure was modified. To avoid homopolymerisation in the water phase the process was divided into two steps. Thanks to the use of two solutions - organic and water - the reagents were separated, which limited the reaction space (free radicals were formed mainly on the polymer/solution interfacial surface) and increased process effectiveness.

In the first step of the process two effects occur: the reagents (CHP, EGDMA) adsorb on the polymer surface and diffuse across the polymer/solution interface, thereafter they accumulate in the thin boundary layer of a polymer matrix. At this stage, time is the limiting factor. The reaction should be long enough for CHP and EGDMA molecules to adsorb. However, its prolongation might lead to their deep penetration inside the polymer. Too deep penetration hinders contact with reagents during the second step of the process and may lead to a potentially toxic effect of the unreacted compounds.

In the second step of the process the adsorbed molecules react with the reagents from water solution (iron ions and PVP), which results in the formation of free radicals and chemical bonds between polymer chains. It should be noted that both CHP and EGDMA are slightly soluble in water. For this reason they only partly dissolve in water, while most of the molecules remain adsorbed on the surface and inside the polymer matrix. Ferrous ions present in the water solution diffuse to the material surface where they catalyse the decomposition of hydroperoxide molecule and the formation of free radicals (Fig. 1, reaction 1). A part of radicals formed from CHP decomposition enters into a reaction with water molecules, in which electrons and hydrogen atoms are intermolecularly transferred. As a result highly reactive hydroxyl radicals are formed (Fig. 1, reaction 3). These radicals are capable of capturing protons from polymer molecules, which leads to formation of macroradicals (Fig. 1, reaction 4). A recombination of macroradicals with the formation of carbon-carbon covalent bonds results in (i) the formation of stable chemical bonds between both the modifying (PVP) and the modified (PU) polymer chains, (ii) the cross-linking of modifying polymer and formation of hydrogel structure (Fig.1, reaction 5). The PVP cross-linking is supported by EGDMA adsorbed on the material surface. EGDMA structure contains double bonds that capture unpaired electrons and form 'bridges' between adjacent polymer chains. A significant role of regeneration of ferrous ions 'used' in the reaction with CHP is played here by ascorbic acid (Fig.1, reaction 2). 


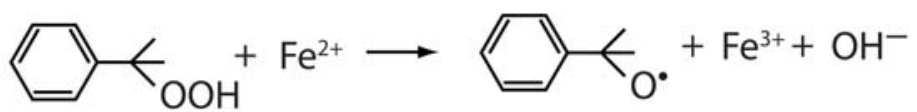

Cumene Hydroperoxide<smiles>[Z7][PH2+][CH+]</smiles>

Ascorbic Acid
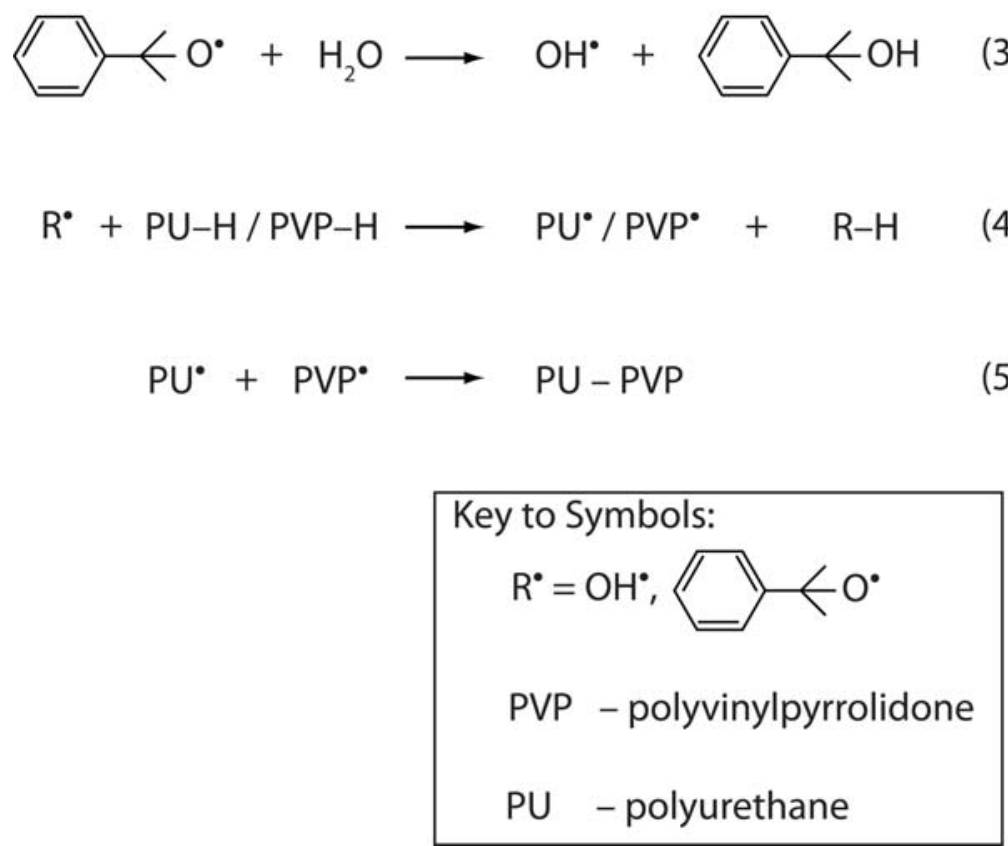

Fig. 1. Radical reactions resulting in formation of hydrogel coating

\subsection{Influence of reagent concentrations on coating properties}

The effect of reagents concentration on the chosen coatings properties was assessed. The equilibrium swelling ratio of a hydrogel coating $(E S R)$ and the mass of hydrogel per surface area $(H R)$ were determined.

In our previous studies (Kaźmierska et al., 2010; Paradowska et al., 2010) we have shown that a PVPbased coating can be obtained without the use of free radicals. The mechanism of this process is based on non-covalent hydrogen bonds formed between hydrogen atoms of polyurethane and oxygen atoms present in PVP molecule. However, such bonds are unstable and a limited number of polymer chains can be bound to the modified layer. In the presented study coatings obtained without the use of free radicals (CHP concentration equals $0 \%$ ) and coatings obtained from solutions containing cumene peroxide (solutions of $2 \%, 4 \%, 8 \%, 12 \%, 16 \% \mathrm{v} / \mathrm{v}$ concentrations) were compared to prove the positive effect of the radical reaction on hydrogel coating properties. The concentrations of other reagents were constant $\left(0.1 \% \mathrm{FeCl}_{2}, 10 \%\right.$ EGDMA, 15\% PVP, $\left.1 \% \mathrm{AA}\right)$. The obtained values of $H G$ and ESR are shown in Fig. 2. 


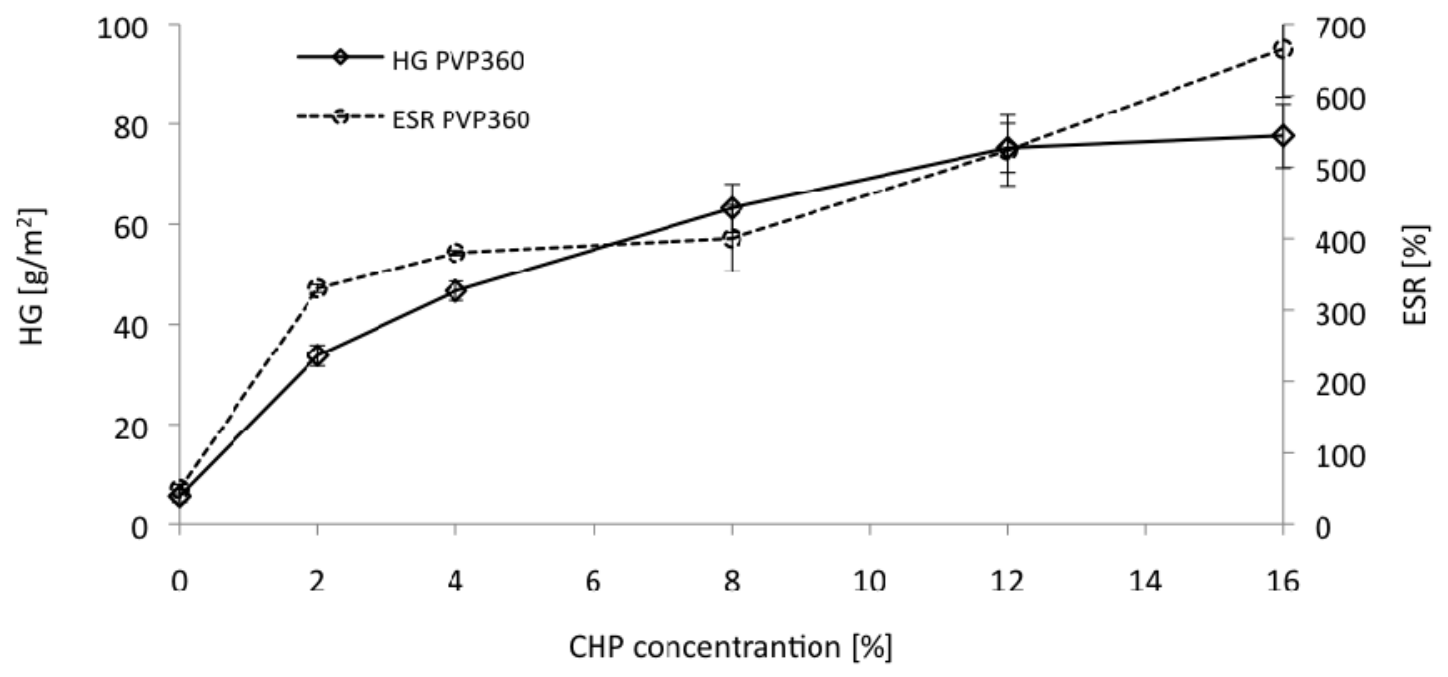

Fig. 2. $H G$ and $E S R$ values for various concentrations $(0 \%, 2 \%, 4 \%, 8 \%, 12 \%, 16 \%)$ of cumene hydroperoxide (CHP) in an organic solution $(M V \pm S D, n=3)$. The concentrations of other reagents were constant and amounted to: $0.1 \% \mathrm{FeCl}_{2}, 10 \%$ EGDMA, $15 \%$ PVP, $1 \% \mathrm{AA}$

As presented in the diagram, $H G$ values are low $\left(H G=5.77 \mathrm{~g} / \mathrm{m}^{2}\right)$ for coatings obtained by the standard procedure without radical reaction. The swelling ratio for these coatings is also low $(E S R=50.14 \%)$. This confirms the fact that coatings obtained without free radical species are unstable and the amount bounded PVP is limited. The introduction of cumene peroxide and the radical formation significantly increased $H G$ and $E S R$ values. Additionally, it was observed that $H G$ and $E S R$ values increased as CHP concentration increased. This correlation is fully understood - the higher the CHP concentration on the material surface, the more free radicals are formed, thereby more PVP chains are bound to material surface. The correlation between CHP concentration and ESR value proves a low degree of hydrogel cross-linking. Thus, it confirms the assumption that radicals are formed mainly on material surface and occur inside the water phase only in a small quantity.

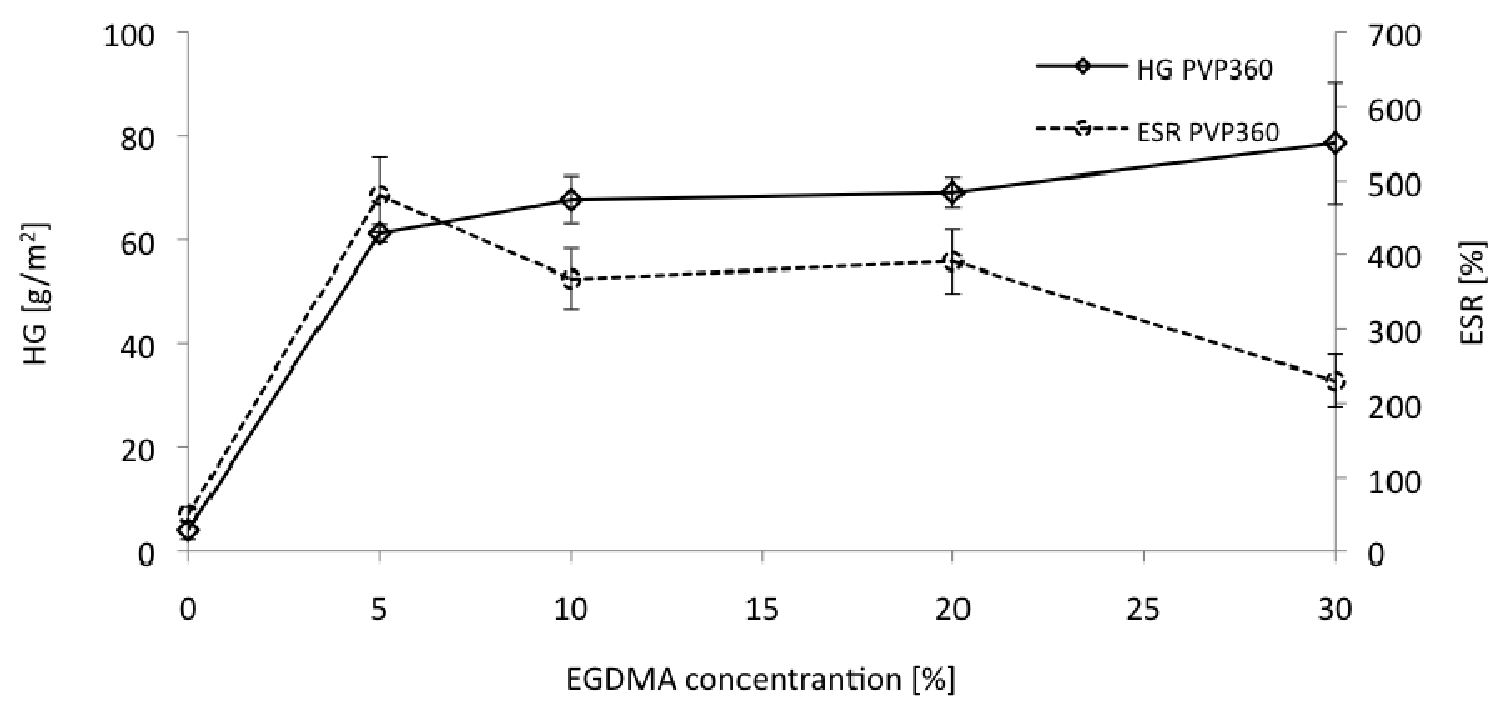

Fig. 3. $H G$ and $E S R$ values for various ethylene glycol dimethacrylate (EGDMA) concentrations ( $0 \%, 5 \%, 10 \%$, $20 \%, 30 \%)$ in an organic solvent $(M V \pm S D, n=3)$. The concentrations of other reagents were constant and amounted to: $0.1 \% \mathrm{FeCl}_{2}, 8 \% \mathrm{CHP}, 15 \% \mathrm{PVP}, 1 \% \mathrm{AA}$ 
The correlation between the evaluated parameters and EGDMA concentration is presented in Fig. 3. A set of solutions with five different EGDMA concentrations $(0 \%, 5 \%, 10 \%, 20 \%$ and $30 \%)$ and a constant concentration of other reagents, namely, $0.1 \% \mathrm{FeCl}_{2}, 8 \% \mathrm{CHP}, 15 \% \mathrm{PVP}$ and $1 \% \mathrm{AA}$ was prepared. In the presented method EGDMA is a hydrogel cross-linking agent. It is noticeable that the presence of EGDMA significantly affects the properties of coatings. At higher EGDMA concentrations the swelling ratio decreases, which proves a high degree of cross-linking. In the absence of crosslinking agent (EGDMA concentration $=0 \%)$ PVP molecules are bound to the material in a limited amount, which results in low $H G$ and ESR values. In the presence of EGDMA, $H G$ values grow significantly; however, a further increase of EGDMA concentration does not affect $H G$ values. As expected, the concentration of cross-linking agent affects mainly the degree of cross-linking and not the coating thickness.

The properties of the coatings are also strongly influenced by ferrous ions concentration in water solution. Five ferrous chloride concentration values were taken into consideration $(0,01 \%, 0,05 \%$, $0,1 \%, 0,5 \%$ and $1 \% \mathrm{w} / \mathrm{v})$. Every solution contained a constant concentration of CHP (8\%), EGDMA (10\%), AA (1\%) and PVP (15\%). The results are presented in Fig. 4. At low $\mathrm{Fe}^{2+}$ concentrations $H G$ and $E S R$ values increase along with the rise of $\mathrm{Fe}^{2+}$ concentration and they reach the highest values for $0.1 \%$ concentration $\left(H G_{\max }=61.69 \mathrm{~g} / \mathrm{m}^{2}\right)$ and $0.05 \%\left(E S R_{\max }=445.60 \%\right)$ respectively. A further increase of $\mathrm{Fe}^{2+}$ concentration results in a significant decrease in the values of both parameters. The possible reasons for this correlation are side reactions presented in Fig. 5. The side reactions are catalysed by $\mathrm{Fe}^{3+}$ ions. At a constant concentration of a reducing agent (ascorbic acid), a higher concentration of $\mathrm{Fe}^{2+}$ ions implicates a higher concentration of $\mathrm{Fe}^{3+}$ ions. $\mathrm{Fe}^{3+}$ ions react with radicals, decreasing the amount of radicals in the system, and therefore reducing the effectiveness of hydrogel forming. Another possible explanation assumes that a high concentration of iron ions provokes fast peroxide decomposition and prevents diffusion of peroxides and radicals into the water phase. Although free radical species are very reactive, they are limited to a narrow space around the polyurethane surface. This results in the formation of thin and highly cross-linked coatings.

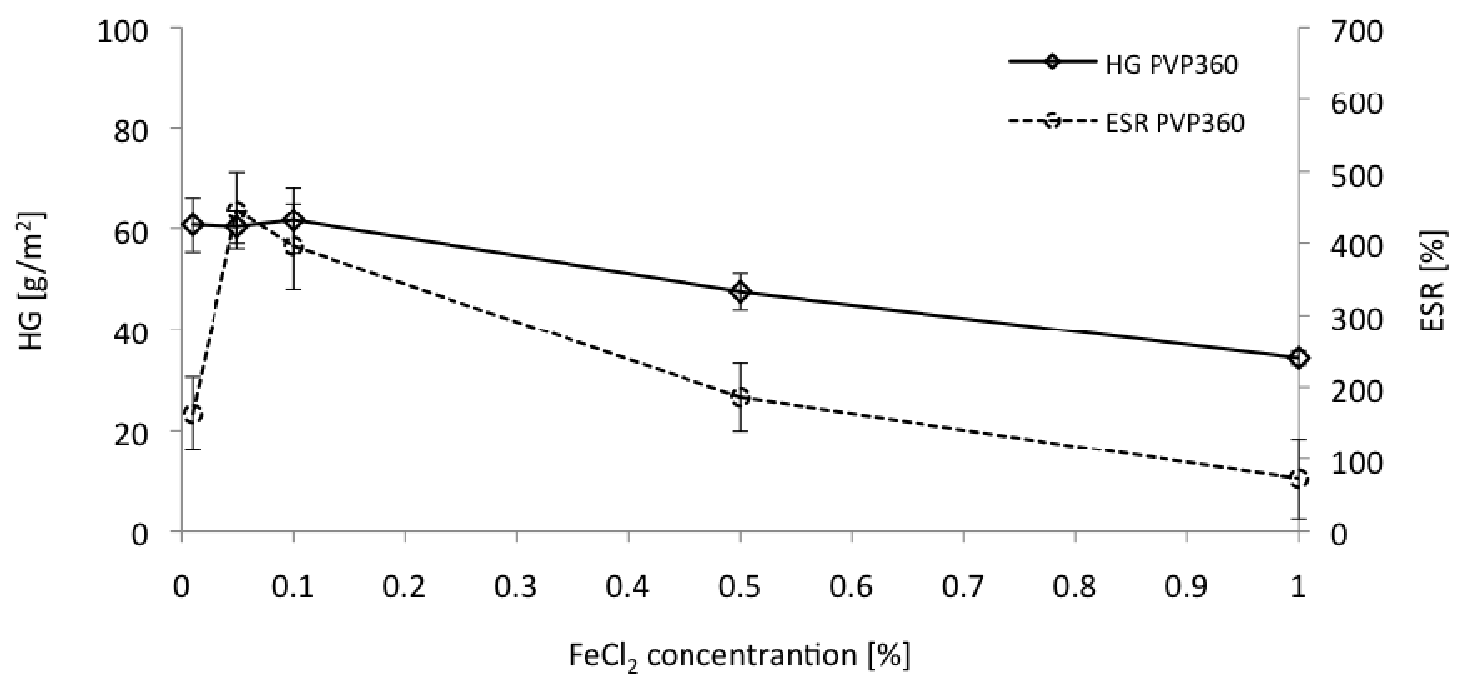

Fig. 4. $H G$ and $E S R$ values for various ferrous chloride $\left(\mathrm{FeCl}_{2}\right)$ concentrations $(0.01 \%, 0.05 \%, 0.1 \%, 0.5 \%, 1 \%)$ in a water solution $(M V \pm S D, n=3)$. The concentrations of other reagents were constant and amounted to: $8 \%$ CHP, $10 \%$ EGDMA, $1 \%$ AA, $15 \%$ PVP

The effect of PVP concentration on coating properties was also assessed. Coatings were obtained using PVP solutions of $0 \%, 5 \%, 10 \%, 15 \%, 20 \%$ and $25 \% \mathrm{w} / \mathrm{v}$ concentrations. The concentrations of other reagents were constant and the values were as follows: $0.1 \% \mathrm{FeCl}_{2}, 8 \% \mathrm{CHP}, 10 \%$ EGDMA and 1\% AA. The results are presented in Fig. 6. It was observed that PVP concentration highly affected the 
thickness of hydrogel coatings. At higher concentrations more PVP molecules bind to the material surface. Interestingly, too high PVP concentration negatively affects coating's durability. As the polymer concentration increases, solution density increases significantly. It prevents diffusion of ferrous ions to the material surface; therefore it reduces the effectiveness of polymerisation. Coatings obtained from solutions containing more than 15\% PVP were poorly bound to the matrix and were easily removed while rinsing. The degree of cross-linking increases with an increase of solution density. It results in a decrease of adsorbing properties of hydrogel and a decrease of ESR values. A significant decrease of ESR was observed for coatings obtained from over 15\% PVP solutions. Furthermore, these coatings showed an unsatisfying quality - too high degree of cross-linking resulted in hydrogel cracking and crushing while drying. The coatings obtained from less concentrated solutions $(5 \%, 10 \%, 15 \%)$ were smooth and strongly bonded to the surface.

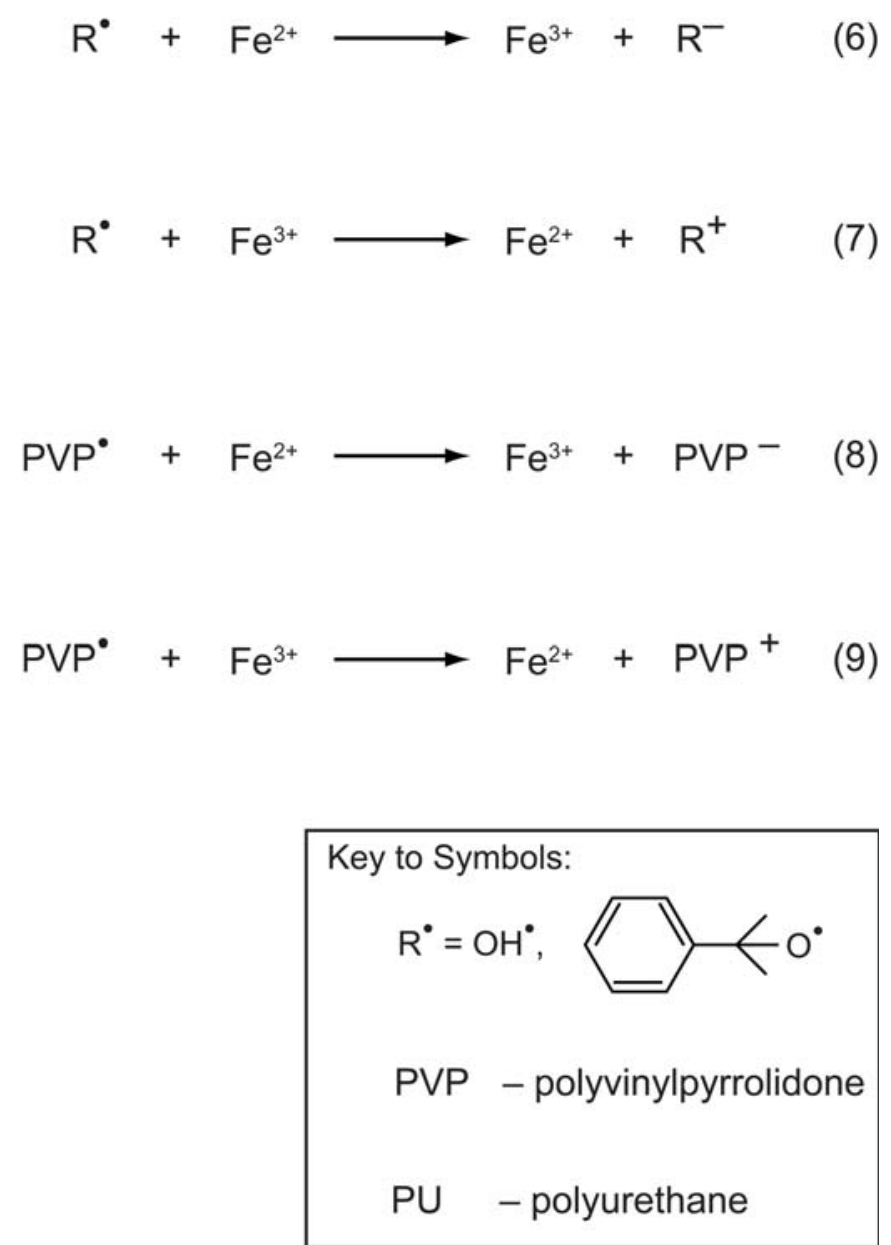

Fig. 5. Side reactions decreasing the amount of free radicals in the system

\subsection{Surface analysis of the coating}

The morphology of modified surfaces was analysed by scanning electron microscopy (SEM) The presence of a highly porous structure, which is typical for hydrogels, was confirmed by SEM pictures (Fig. 7a: SEM image of a sample prepared from solutions containing $0.1 \% \mathrm{FeCl}_{2}, 5 \% \mathrm{CHP}, 10 \%$ EGDMA, $10 \%$ PVP and 1\% AA). 


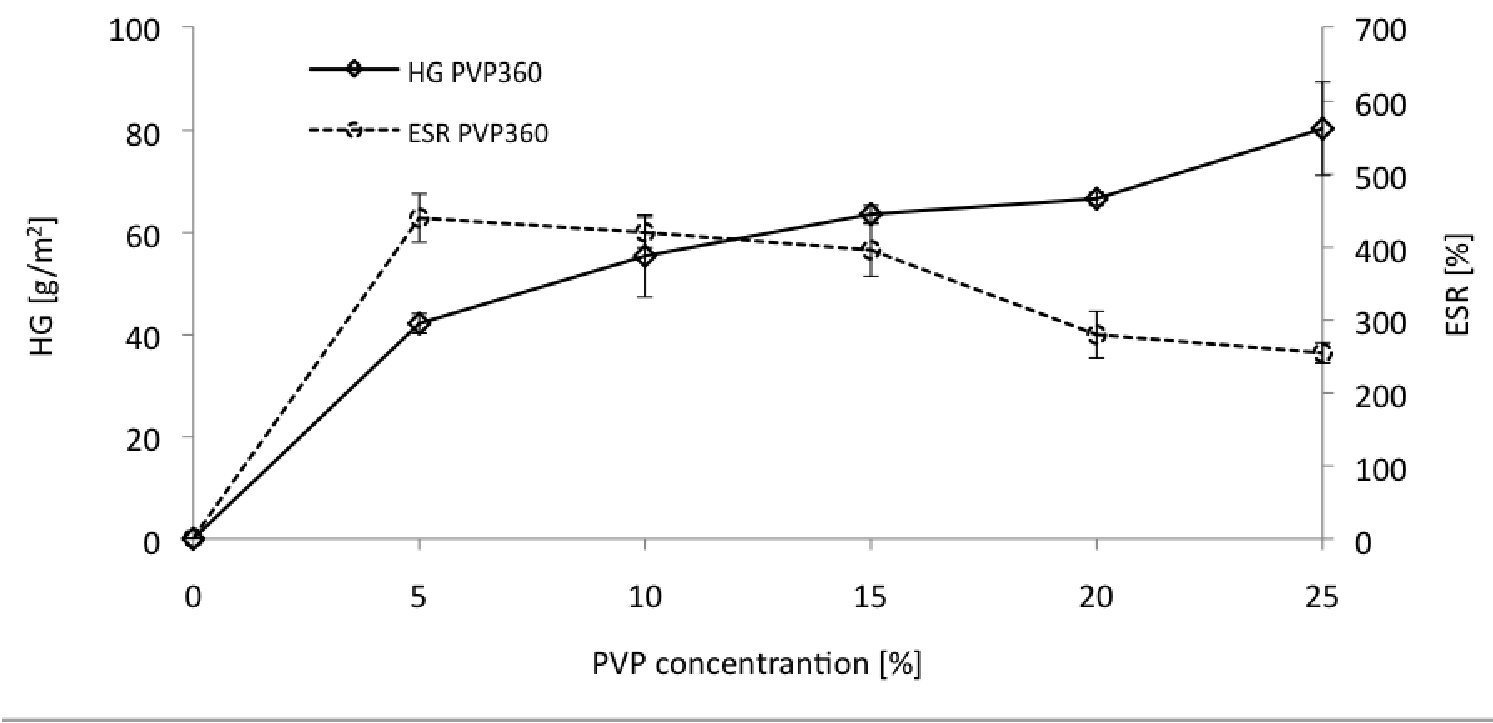

Fig. 6. $H G$ and $E S R$ values for various PVP concentrations $(0 \%, 5 \%, 10 \%, 15 \%, 25 \%)$ in a water solution $(M V \pm S D, n=3)$. The concentrations of other reagents were constant and amounted to: $0.1 \% \mathrm{FeCl}_{2}, 8 \% \mathrm{CHP}$, $10 \%$ EGDMA and $1 \%$ AA
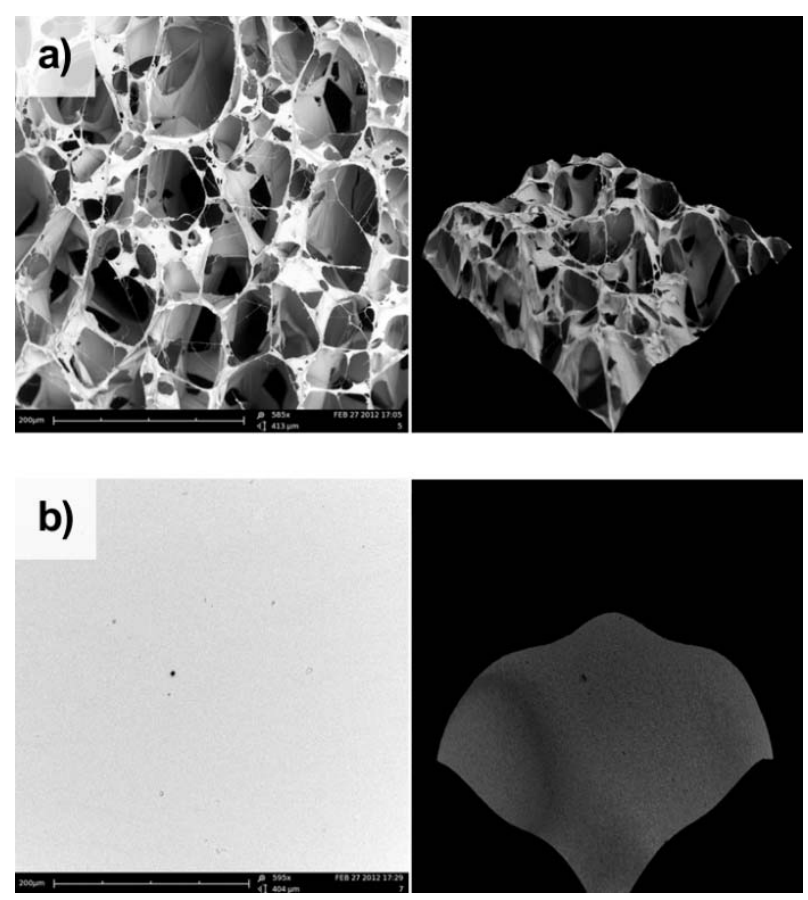

Fig. 7. 2D (left) and 3D (right) SEM images of hydrogel-coated polyurethane (a) and uncoated polyurethane (b)

\section{CONCLUSIONS}

In this paper we proposed a simple method of coating polyurethane materials with hydrogel layer. The modification is based on free radical macromolecular grafting-crosslinking. The fabricated coating is covalently bonded to the polymer substrate, which assures a proper durability. The presented results showed that concentrations of all the reagents used in the fabrication process strongly influences the physical properties of the obtained hydrogel layers. The coating was designed to increase hydrophilicity of the implantable polymer materials. Both polymers applied in the presented technology, PU and PVP, are highly biocompatible and widely used in biomedical applications. Thus, the presented technology can be used to produce biocompatible surfaces with limited protein and cell adhesive properties and can 
be applied in the fabrication of a number of biomedical devices, e.g. catheters, vascular grafts and heart prosthesis.

The presented work has been supported by the Polish Artificial Heart Project and the European Union in the framework of European Social Fund through the Warsaw University of Technology Development Programme. Patent applications for the presented coating technology are pending.

\section{SYMBOLS}

ESR equilibrium swelling ratio, \%

$H G \quad$ mass of hydrogel grafted to the surface per surface area, $\mathrm{g} / \mathrm{m}^{2}$

PU polyurethane

PVP poly (vinyl pirrolidone)

SEM scanning electron microscope

\section{REFERENCES}

Butruk B., Trzaskowski M., Ciach T., 2012. Fabrication of biocompatible hydrogel coatings for implantable medical devices using Fenton-type reaction. Mat. Sci. Eng.: C., 32, 1601-1609. DOI: 10.1016/j.msec.2012.04.050.

Kaźmierska K., Szwast M., Ciach T., 2010. Determination of urethral catheter surface lubricity. J. Mater. Sci. Mater., 19, 2301-2306. DOI: 10.1007/s10856-007-3339-4.

Kim J.H., Kim S.C., 2002. PEO-grafting on PU/PS for enhanced blond compatibility - effect of pendant length and grafting density. Biomater., 23, 2015-2025. DOI: 10.1016/S0142-9612(01)00330-1.

Le Y., Scott M., 2010. Immunocamouflage: The biophysical basis of immunoprotection by grafted methoxypoly(ethylene glycol) (mPEG). Acta Biomater., 6, 2631-2641. DOI: 10.1016/j.actbio.2010.01.031.

Patel A, Mequanint K., 2007. Novel physically crosslinked polyurethane-block-poly(vinyl pyrrolidone) hydrogel biomaterials. Macromol. Biosci., 7, 727-737. DOI: 10.1002/mabi.200600272.

Paradowska A.E., Kaźmierska K.A., Ciach T., 2010. Influence of the coating process parameters on the quality of PUR/PVP hydrogel coatings for PVC medical devices. Pol. J. Chem. Technol., 12, 38-45. DOI: 10.2478/v10026-010-0016-z.

Walling C., El-Taliawi G.M., Johnson R.A., 1974. Fenton's reagent. IV. Structure and reactivity relations in the reactions of hydroxyl radicals and the redox reactions of radicals, J. Am. Chem. Soc., 96, 133-139. DOI: $10.1021 / \mathrm{ja} 00808 \mathrm{a} 022$.

Xu L., Siedlecki C.A., 2007. Effects of surface wettability and contact time on protein adhesion to biomaterial, Biomater., 28, 3273-3283. DOI: 10.1016/j.biomaterials.2007.03.032. 